\title{
BMJ Open Eating Disorder Neuroimaging Initiative (EDNI): a multicentre prospective cohort study protocol for elucidating the neural effects of cognitive-behavioural therapy for eating disorders
}

To cite: Hamatani S, Hirano Y, Sugawara A, et al. Eating Disorder Neuroimaging Initiative (EDNI): a multicentre prospective cohort study protocol for elucidating the neural effects of cognitive-behavioural therapy for eating disorders. BMJ Open 2021;11:e042685. doi:10.1136/ bmjopen-2020-042685

- Prepublication history and additional material for this paper is available online. To view these files, please visit the journal online (http://dx.doi.org/10. 1136/bmjopen-2020-042685).

Received 11 July 2020 Revised 09 December 2020 Accepted 10 January 2021

Check for updates

(c) Author(s) (or their employer(s)) 2021. Re-use permitted under CC BY-NC. No commercial re-use. See rights and permissions. Published by BMJ.

For numbered affiliations see end of article.

Correspondence to Dr Atsushi Sekiguchi; asekiguchi@ncnp.go.jp

\section{ABSTRACT}

Introduction Anorexia nervosa is a refractory psychiatric disorder with a mortality rate of $5.9 \%$ and standardised mortality ratio of 5.35 , which is much higher than other psychiatric disorders. The standardised mortality ratio of bulimia nervosa is 1.49; however, it is characterised by suicidality resulting in a shorter time to death. While there is no current validated drug treatment for eating disorders in Japan, cognitive-behavioural therapy (CBT) is a well-established and commonly used treatment. CBT is also recommended in the Japanese Guidelines for the Treatment of Eating Disorders (2012) and has been covered by insurance since 2018. However, the neural mechanisms responsible for the effect of CBT have not been elucidated, and the use of biomarkers such as neuroimaging data would be beneficial.

Methods and analysis The Eating Disorder Neuroimaging Initiative is a multisite prospective cohort study. We will longitudinally collect data from 72 patients with eating disorders (anorexia nervosa and bulimia nervosa) and 70 controls. Data will be collected at baseline, after 21-41 sessions of CBT and 12 months later. We will assess longitudinal changes in neural circuit function, clinical data, gene expression and psychological measures by therapeutic intervention and analyse the relationship among them using machine learning methods.

Ethics and dissemination The study was approved by The Ethical Committee of the National Center of Neurology and Psychiatry (A2019-072). We will obtain written informed consent from all patients who participate in the study after they had been fully informed about the study protocol. All imaging, demographic and clinical data are shared between the participating sites and will be made publicly available in 2024 . Trial registration number UMIN000039841

\section{INTRODUCTION}

Eating disorders (EDs) are a psychiatric disorder with a focus on body shape, weight
Strengths and limitations of this study

The research project will seek to clarify diagnostic and therapeutic markers and identify predictive markers of treatment response in patients with eating disorders using a longitudinal approach.

- This should contribute to the early detection and early intervention of eating disorders.

- We will also create a brain imaging database in Japan for those with eating disorders.

- MRI equipment and treatment protocols are not completely unified.

and abnormal eating behaviours. They are mainly classified as anorexia nervosa (AN), bulimia nervosa (BN) and binge-eating disorder (BED). ${ }^{1}$ In Japan, the number of patients with EDs has increased about 10-fold between the 1980s and 1990s. ${ }^{2}$ Based on a meta-analysis including 36 studies, AN was found to have a standardised mortality ratio (SMR) of $5.1^{3}$ and the highest mortality rate among mental disorders. ${ }^{4-7}$ In addition, renal function decreases as the duration of AN increases, ${ }^{8}$ and patients often suffer from various physical complications associated with prolonged symptoms and low weight. ${ }^{9-12}$ The rate of chronicity for patients with AN for up to 10 years is approximately $10 \%-20 \%$, and the long-term prognosis is poor. ${ }^{13}{ }^{14}$ Furthermore, current treatments may not be effective in severe cases of chronic AN. ${ }^{15}$ Regarding BN, the SMR is 1.49 ; however, it is characterised by suicidality resulting in a shorter time to death. ${ }^{3}$ 
Therefore, early detection and early intervention are important.

Cognitive-behavioural therapy (CBT) is a psychotherapy that aims to improve psychiatric symptoms by focusing on cognitive processes and behavioural patterns. CBT for EDs identifies the psychopathology that contributes to the disease while advancing regular eating and weight-regulating behavioural modifications. ${ }^{16}$ Cognitive styles such as fear of weight gain and adherence to body shape and behavioural patterns such as dietary restrictions and excessive exercise are often seen in $\mathrm{EDs}^{17}$; CBT can be used to address these psychopathologies. ${ }^{16}$ CBT has been shown to be effective as a psychotherapy for EDs, ${ }^{18-20}$ and CBT is also recommended in the National Institute for Health and Care Excellence (NICE) guidelines. ${ }^{21}$ Meta-analyses of Randomised Controlled Trials (RCTs) that include waitlist controls report that CBT is particularly effective for BN and BED. ${ }^{22-26}$ In Japan, there are two reports on the effectiveness and feasibility of CBT for $\mathrm{BN}$ or $\mathrm{BED},{ }^{27} 28$ and the national health insurance has covered CBT in Japan since 2018. However, according to a meta-analysis of RCTs, the dropout rate for CBT is approximately $24 \%,{ }^{29}$ and there are large individual differences in treatment responsiveness.

To the best of our knowledge, there are no reports of neural mechanisms that regulate the effects and treatment responses of CBT for EDs. Therefore, accumulating evidence using brain imaging and biomarkers of gene expression would further our understanding of CBT effectiveness for EDs. The identification of biomarkers may provide clues to the development of diagnostic and therapeutic methods based on the elucidation of the pathophysiology and pathogenic mechanism of EDs. It may contribute to social implementation of early detection and early intervention with diagnostic methods using objective indicators.

Since individuals with EDs show abnormal eating behaviours, structural and functional brain imaging studies have been conducted to elucidate the neural basis of these abnormalities through brain imaging research. There are various reports on brain imaging research in patients with ED that have revealed abnormal reward systems, ${ }^{30}$ reductions in grey matter of various brain regions (frontal lobes, ${ }^{31}$ frontal gyrus, ${ }^{32}$ parietal and temporal lobes, ${ }^{33}$ occipital lobe ${ }^{34}$ ) and limited reductions in white matter volume. ${ }^{35}$ In Japan, the neural basis for the pathogenesis of EDs has been studied. ${ }^{36-39}$ However, brain imaging research for EDs has only been conducted in the form of small cross-sectional studies in a single facility, and the reproducibility and validity of these results remain questionable. Therefore, research at a larger scale is needed to elucidate the cognitive brain science of ED pathology and progression and to identify clinically useful diagnostic and therapeutic markers and prognostic predictors.

The ENIGMA-Eating Disorders should be noted as a multicentre study outside of Japan. However, it is only registered by researchers in Europe, the USA and Africa and mainly takes place in Germany. For the time being, they have used methods to meta-analyse anatomical images and diffusion tensor-weighted images of patients with $\mathrm{AN}$ and $\mathrm{BN}$ that have already been imaged at participating facilities. The ENIGMA-Eating Disorders study is expected to build a framework for joint research to be developed in the future. However, ED brain image multicentre research has not been found to have been conducted, even when searching the world's major clinical research registration sites such as ClinicalTrials.gov in the USA and EU register in the EU. Furthermore, an ED brain image database has not yet been developed globally.

\section{Aims and hypotheses}

We aim to generate neuroscientific evidence for the effect of CBT. First, we will longitudinally collect brain MRI images and clinical data in observational studies before and after CBT for EDs. Next, we will identify clinical biomarkers of EDs through analytical studies using longitudinal image data before and after CBT for EDs and create neural evidence for the effect of CBT. We aim to identify brain image biomarkers that can be used as clinical markers (diagnostic markers, therapeutic markers and therapeutic response prediction markers) for EDs. We hypothesise the following: (1) characteristic brain circuit abnormalities exist for each type of ED; (2) there are brain circuit changes that correlate with changes in severity before and after treatment for ED; and (3) there are brain circuit features that define the therapeutic response of CBT for ED. Furthermore, the brain image data collected for this project will be integrated into an international brain database prepared by the Japan Agency for Medical Research and Development (AMED). In the future, after the AMED Brain/MINDS Beyond human brain MRI database is constructed, it will be possible to access the database. At the moment, the portal site (https://brainminds-beyond.jp/ja/resources/ 2020/05/amedmri.html) is being created (Details of The Brain / MINDS Beyond human brain MRI project: Koike et al, 2020 preprint).

\section{METHODS AND ANALYSIS}

\section{Study design}

This is a multisite, observational cohort study. For patients with $\mathrm{ED}$ who have received structured $\mathrm{CBT}$, the following will be performed before and after CBT: brain MRI (T1-weighted image (T1WI), T2-weighted image (T2WI), resting state functional MRI and diffusion tensor imaging (DTI)); blood collection for gene polymorphism and gene expression analysis; and psychological evaluation.Table 1 shows the study design. In the pretreatment evaluation, subjects will visit each treatment facility for treatment, and a doctor or psychologist (recruiter) will evaluate eligibility and exclusion criteria for CBT introduction. Among the subjects who meet the CBT eligibility criteria, the eligibility criteria and exclusion criteria of this study will be evaluated. Subjects who have obtained informed consent for this study will be registered as study 
Table 1 Standard protocol items

\begin{tabular}{|c|c|c|c|c|}
\hline Item & Pre & Intervention & Post & Follow-up \\
\hline Time point & $-4 W-0$ & $1 \sim 21$ sessions $^{*}$ or 41 sessionst & Within 4 weeks & $50 \pm 8 W$ \\
\hline Informed consent & $x$ & & - & - \\
\hline EDE 17.0D & $x$ & & $x$ & $x$ \\
\hline EDE-Q & $\times$ & & $\times$ & $\times$ \\
\hline BDI-II & $x$ & & $x$ & $x$ \\
\hline STAI & $\times$ & & $\times$ & $\times$ \\
\hline NEO-FFI & $x$ & & $x$ & $x$ \\
\hline TAS-20 & $x$ & & $x$ & $x$ \\
\hline PDS & $x$ & & - & - \\
\hline VAS & $x$ & & $x$ & $x$ \\
\hline GF-FAD & $x$ & & $x$ & $x$ \\
\hline MAIA & $x$ & & $\times$ & $\times$ \\
\hline BIS-11 & $x$ & & $x$ & $x$ \\
\hline $\mathrm{MOCl}$ & $\times$ & & $\times$ & $\times$ \\
\hline$A Q$ & $x$ & & - & - \\
\hline SES & $x$ & & $\times$ & $x$ \\
\hline TAC24 & $x$ & & $x$ & $x$ \\
\hline Socioeconomic status & $\times$ & & - & - \\
\hline SSS & $x$ & & $x$ & $x$ \\
\hline Brain MRI examination & $x$ & & $x$ & $x$ \\
\hline Blood sample (non-fasting period) & $x$ & & $x$ & $x$ \\
\hline
\end{tabular}

*Outpatient.

†Inpatient.

AQ, Autism-Spectrum Quotient; BDI-II, Beck Depression Inventory-Second Edition; BIS-11, Barratt Impulsiveness Scale; CTQ, Childhood Trauma Questionnaire for childhood trauma; EDE 17.0D, Eating Disorder Examination Edition 17.0D; EDE-Q, Eating Disorders Examination Questionnaire; EQ-5D, EuroQol-5 Dimension; GAD-7, Generalized Anxiety Disorder Assessment; GF-FAD, General Functioning scale of Family Assessment Device; JART, Japanese Adult Reading Test; MAIA, Multidimensional Assessment of Interoceptive Awareness; M.I.N.I, Mini-International Neuropsychiatric Interview; MOCI, Maudsley Obsessional Compulsive Inventory; NEO-FFI, NEO Five-Factor Inventory; PDS, Post-traumatic Diagnostic Scale for Trauma; PHQ-9, Patient Health Questionnaire; SES, Rosenberg Self-Esteem Scale; SSS, Stanford Sleepiness Scale; STAI, State-Trait Anxiety Inventory; TAC24, Tri-axial Coping Scale; TAS20, 20-Item Toronto Alexithymia Scale; VAS, Visual Analogue Scale; WAI, Working Alliance Inventory; WHO-5, WHO - Five Well-Being Index.

subjects. After registration, the schedule for the first session of CBT will be determined. In principle, brain MRI scans and other tests will be carried out within 4 weeks before the session date. The post-treatment evaluation is based on the day when CBT for EDs is completed, and in principle, tests such as brain MRI are performed within 4 weeks. In addition, a similar assessment 1 year after treatment ( $50 \pm 8$ weeks) will be performed for subjects who are willing.

\section{Study setting}

This trial will be conducted by seven facilities (National Center of Neurology and Psychiatry (NCNP), Chiba 
University, Tohoku University, Tokyo University, Kyoto University, Kyushu University and University of Occupational and Environmental Health) in Japan.

\section{Recruitment}

Those who wish to participate in the study will visit the NCNP, Chiba University, Tohoku University, Tokyo University, Kyoto University, Kyushu University and University of Occupational or Environmental Health. The study participants will be recruited through posters and leaflets placed, through the official web-based advertisements, and by referrals from their primary care doctors or psychiatrists to receive CBT treatment. The doctor or psychologist (recruiter) evaluates the eligibility criteria and exclusion criteria for CBT introduction. Among the subjects who meet the CBT eligibility criteria, the eligibility criteria and exclusion criteria of this study will be evaluated. Subjects who have obtained informed consent for this study will be registered as subjects for this study.

\section{Study participants}

Patients will be enrolled in the study if they meet the following selection criteria without meeting the exclusion criteria (see table 2).

\section{Patient and public involvement}

Patients and public were not involved in the design of this study.

\section{Interventions}

In principle, CBT 'improved version, ${ }^{20}$ for EDs, 20-40 session version will be implemented (UMIN000031625). However, structured CBT performed at each treatment facility is acceptable (UMIN000039485, UMIN000036825 and so on). Therapists are specialists (psychiatrists and certified public psychologists) who have attended workshops and have been trained in conducting CBT. In addition, each CBT is manualised and structured. Treatment compliance/fidelity is basically maintained by each therapist receiving supervision and following manuals.

\section{Primary outcome}

The primary outcome was ED symptoms and severity assessed by the Eating Disorder Examination Edition 17.0D (EDE 17D) ${ }^{40} 41$ or Eating Disorders Examination Questionnaire (EDE-Q) ${ }^{41}{ }^{42}$ The EDE 17D is a semistructured interview, whereas the EDE-Q is a self-contained, 28-item questionnaire derived from the Eating Disorder Examination Edition (EDE). The EDE-Q is scored on a 7-point Likert scale (0-6) on which a score of $\geq 4$ indicates a clinical range. The global score on the EDE-Q is the sum of the four subscale scores (for restraint, eating concern, shape concern and weight concern) divided by 4 .

\section{Secondary outcomes}

1. Remission at the end of treatment (state that does not meet DSM-5 criteria).

2. Treatment completion rate (completed when $75 \%$ or more of 21-41 treatment sessions are received).
3. ED-specific indicators: current BMI; the lowest and highest BMI in the past; history of $\mathrm{AN}$ and $\mathrm{BN}$.

4. Non-specific psychological indicators (see details as a online supplemental file 1): the Mini-International Neuropsychiatric Interview ${ }^{43}{ }^{44}$ for comorbidities; Japanese Adult Reading Test ${ }^{45}{ }^{46}$ for intellectual ability; Beck Depression Inventory-Second Edition ${ }^{47} 48$ for severity of depressive symptoms; State-Trait Anxiety Inventory $^{49}{ }^{50}$ for anxiety; NEO Five-Factor Inventory $(\mathrm{NEO}-\mathrm{FFI})^{51}{ }^{52}$ for personality scores; 20-Item Toronto Alexithymia Scale ${ }^{53}{ }^{54}$ for alexithymia; adult version facial expression recognition test ${ }^{55}$ for adult facial expression recognition ability; Edinburgh handedness inventory ${ }^{5657}$ for determining objectively whether one is left or right handed; Childhood Trauma Questionnaire ${ }^{58}$ for childhood trauma; Post-traumatic Diagnostic Scale ${ }^{6061}$ for trauma; Visual Analogue Scale ${ }^{62}$ for expectation to treatment; General Functioning scale of Family Assessment Device ${ }^{63}$ for family assessment; help-seeking preferences ${ }^{65}$ for attitude to seek help for others; Multidimensional Assessment of Interoceptive Awareness ${ }^{66} 67$ for relevant dimensions of bodily awareness; Barratt Impulsiveness Scale ${ }^{68}$ for impulsivity scale; Maudsley Obsessional Compulsive Inventory $^{70}{ }^{71}$ for obsessive-compulsive symptoms scale; Autism-Spectrum Quotient ${ }^{72}{ }^{73}$ for autism tendencies; Rosenberg Self-Esteem Scale (SES) ${ }^{74}$ for global selfesteem; Tri-axial Coping Scale ${ }^{76}$ for evaluation of stress coping strategies; Working Alliance Inventory ${ }^{77}$ for aspects of the therapeutic alliance; EuroQol-5 Dimension $^{78} 79$ for quality of life; the Generalized Anxiety Disorder Assessment ${ }^{80} 81$ for severity of anxiety; Patient Health Questionnaire ${ }^{81} 82$ for depression module; the WHO - Five Well-Being Index ${ }^{83}$ for mental health scale; socioeconomic status for education history; and Stanford Sleepiness Scale ${ }^{84}$ for subjective sleepiness levels.

5. Brain image data, gene polymorphism/gene expression analysis data.

\section{DEMOGRAPHIC DATA}

At the time of pre-CBT, we will collect the following demographic data: age, educational background, marital status, cohabitation/family/presence of partner, hospitalisation history, age of onset, medical history, comorbidities, family history and medication content.

\section{Imaging acquisition}

MRI scans will be obtained in all participants on 3 Tesla scanners: Siemens MAGNETOM Prisma (University of Tokyo), Skyrafit (NCNP) and Verio (Kyoto University); GE Discovery MR750 3.0T (Chiba University), Premier (University of Occupational and Environmental Health); and Phillips Ingenia 3.0T CX (Tohoku University and Kyushu University). The brain MRI examination takes T1WI, T2WI, resting-state fMRI and diffusion tensorweighted images. The imaging protocol is according to Harmonized Protocol (HARP) ${ }^{85}$ for clinical MRI studies 


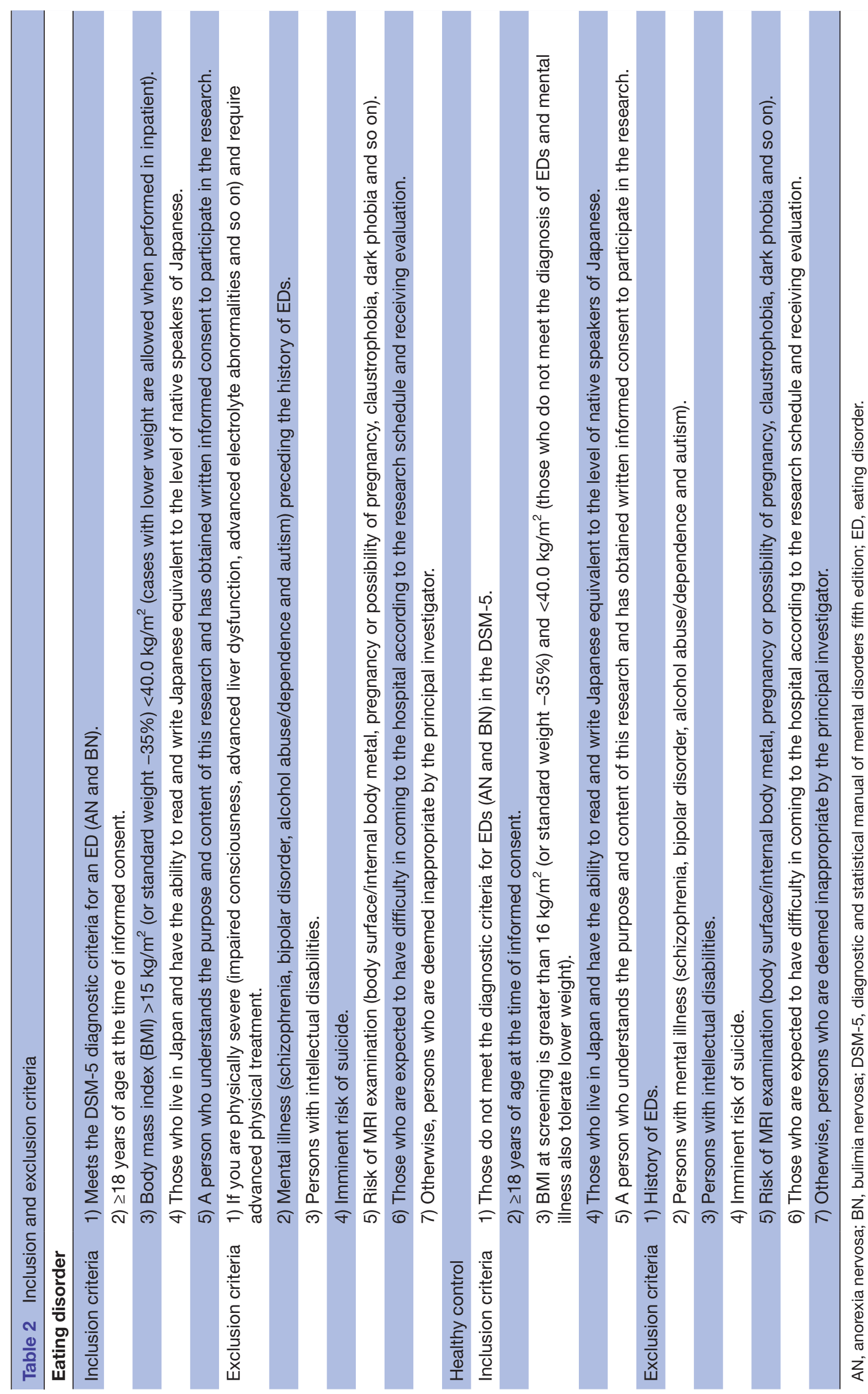




\begin{tabular}{|c|c|c|c|c|c|c|c|}
\hline Institution & THK & CHB & UTI & NCNP & KYU & UOEH & KYS \\
\hline MRI site & IDAC & CHB2 & UTI2 & NCNP2 & $\mathrm{KRC2}$ & OEH & KYS \\
\hline $\begin{array}{l}\text { Number of } \\
\text { channels per } \\
\text { coil }\end{array}$ & 20 & 32 & 32 & 32 & 32 & 48 & 20 \\
\hline $\begin{array}{l}\text { Imaging } \\
\text { protocol }\end{array}$ & SRPB & SRPB & HARP & HARP & HARP & SRPB & SRPB \\
\hline
\end{tabular}

*Institutions that originally planned for SRPB may replace after HARP was developed.

CHB, Chiba University; HARP, Harmonized Protocol; IDAC, Institute of Development Aging and Cancer; KRC, Kokoro Research Center; KYS, Kyushu University; KYU, University of Kyoto; NCNP, National Center of Neurology and Psychiatry; SRPB, Strategic Research Program for Brain Science; THK, Tohoku University; UOEH/OEH, University of Occupational and Environmental Health; UTI, The University of Tokyo IRCN.

in Brain/MINDS Beyond when multiband echo-planar imaging option is enabled in each site. If not, the imaging protocol will be adopted based on the Strategic Research Program for Brain Science (SRPB) protocol. Resting-state fMRI will be acquired in an open-eye condition. MRI model, coil and imaging parameters are shown in tables 3 and 4 .

\section{Biological measures from blood samples}

For blood tests, approximately $20 \mathrm{~mL}$ of blood will be collected at a time, with a total of approximately $60 \mathrm{~mL}$ during the study period. For shock symptoms due to vasovagal reactions, physical condition will be checked on the day of blood collection, and patients will be closely observed for $5 \mathrm{~min}$ after the start of blood collection when this reaction is likely to occur. Blood samples will include blood plasma, serum, blood sampling for DNA methylome and blood sampling for RNA transcriptomics.

\section{Data logistics}

The brain imaging data collected at all facilities will be anonymised at each facility and then aggregated in the NCNP through the Integrative Brain Imaging Support System. The primary analysis will be performed in the NCNP. The results of the primary analysis will be shared by all facilities. Blood samples collected at all facilities will be anonymised, and all samples for gene polymorphism/ gene expression analysis will be sent to NCNP where the samples will be extracted and stored in a $-80^{\circ} \mathrm{C}$ freezer. As soon as approximately 20 cases are collected, the gene expression analysis will be outsourced to a contractor. In addition, psychological and clinical data will be anonymised at each institution and then shared by all institutes (see the figure 1). The correspondence table for all data will be managed at the facility where the data were collected.

\section{Statistical analysis}

Brain imaging data collected at all facilities are subjected to a primary analysis by the analysis pipeline from NCNP. From the brain images of each individual, the data representing brain grey matter mass, white matter integrity and resting functional connection of the region of interest will be extracted. In addition, we will evaluate functional connectivity in various intracerebral networks and between networks such as the default mode network, salience network, dorsal attention network, cognitive control network and affective network in resting brain activity. For example, we plan to perform seed-based analysis with CONN toolbox (www.nitrc.org/projects/ conn) ${ }^{86}$ We plan to verify the presence or absence of network abnormalities using the orbitofrontal cortex and anterior cingulate cortex, which have been pointed out as seeds in ED, and whether these network abnormalities have changed after CBT.

At the secondary analysis stage, strategies (harmonisation) for adjusting interfacility factors will be considered. At present, we are planning to use corrections based on average values between facilities or software such as Combat. The ComBat harmonisation tool ${ }^{87-89}$ uses Bayesian regression to find systematic differences among multiple data collected using different scanners. The tool performs additive and multiplicative corrections to produce distortions that eliminate these systematic differences from the data; however, as new harmonisation strategies are developed, they will be tested as appropriate. At the time when about 20 longitudinal data are collected, the data will be fixed after being integrated with psychological/clinical data other than brain images and gene polymorphism/gene expression analysis results, and the following analysis will begin: (1) identification of diagnostic markers (baseline data 20 people): compare clinical symptoms and brain images, psychology, gene polymorphism and gene expression data at baseline in $\mathrm{AN}$ and $\mathrm{BN}$ disease types, and search for cognitive, psychological and behavioural indicators associated with disease type diagnosis. At this time, a comparison with the healthy group will also be performed. By comparing these groups, diagnostic markers based on conventional diagnoses will be identified (categorical approach). Furthermore, we will identify diagnostic markers on searching 


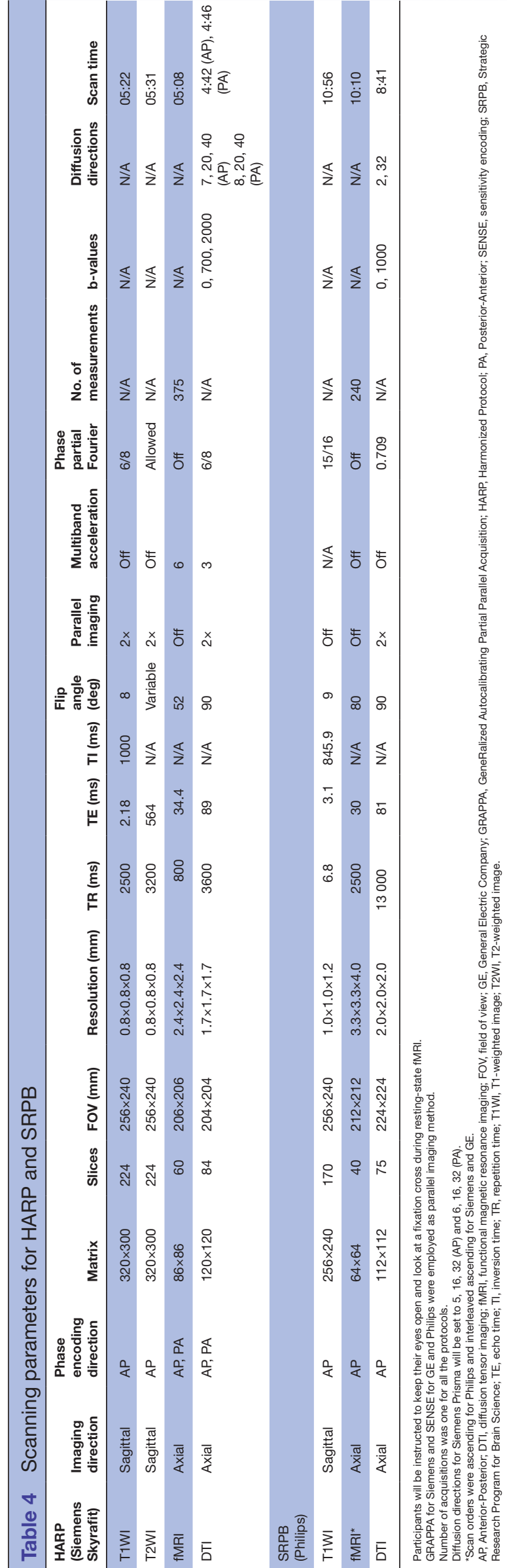

for psychopathological features in brain images, gene polymorphisms and gene expression data related to core disease state indicators and other psychological indicators across all disease types (dimensional approach); (2) identification of therapeutic effect (longitudinal data set 40 people): we will analyse the relationship between changes in ED symptoms before and after $\mathrm{CBT}$ and changes (rates) in brain images, psychology, gene polymorphisms and gene expression data, and identify the therapeutic effects of CBT; (3) identification of therapeutic response markers (longitudinal data set 60): analysing associations with baseline brain images, psychology, gene polymorphisms and gene expression data to predict the completion and remission of EDs before and after CBT, and identify treatment predictive markers; (4) in addition to the above, analysis that is judged necessary or meaningful at the time of analysis will be performed. In particular, we plan to conduct analysis using machine learning techniques. If there are missing values in the dataset to be analysed, they will, in principle, be excluded from the analysis.

\section{Machine-learning approach}

We are planning to use the python Scikitlearn library (V.0.22.1) to perform support vector machine (SVM) classification. ${ }^{90}$ To prevent overfitting (ie, the classifier works perfectly on the training data but is poorly generalisable to new data), we will perform a feature relevance evaluation and dimensionality reduction using a tree-based feature selection approach with a nested cross-validation design. ${ }^{91-93}$

The nested cross-validation consists of an inner loop for model building and parameter estimation and an outer loop for model testing. Consequently, the dataset will be divided into two parts: a training plus validation subset and a test subset. In the inner loop, SVM models will have been trained with varying SVM hyperparameters (ie, cost parameters $\mathrm{C}$ and kernel function) based on a grid search, and a feature selection will have been performed using classification trees or random forests. The validation set will be used to determine the SVM hyperparameters over the grid of possible values. The performance of the resulting model, with optimised SVM hyperparameters and features, will be subsequently evaluated on the test set in the outer loop. For this outer loop, we will use a leave-one-out cross-validation scheme so that each sample will be used once as a test set (singleton) while the remaining samples form the training/validation set. The training/validation set will be divided into five equally sized parts. Four of these will be used as the training set and one as the validation set. This process is repeated until all subjects are left out at least once and prediction results are aggregated as well as reported.

\section{SAMPLE SIZE}

The number of cases needed to detect an improvement in ED symptoms, which is the main outcome, was calculated. If 


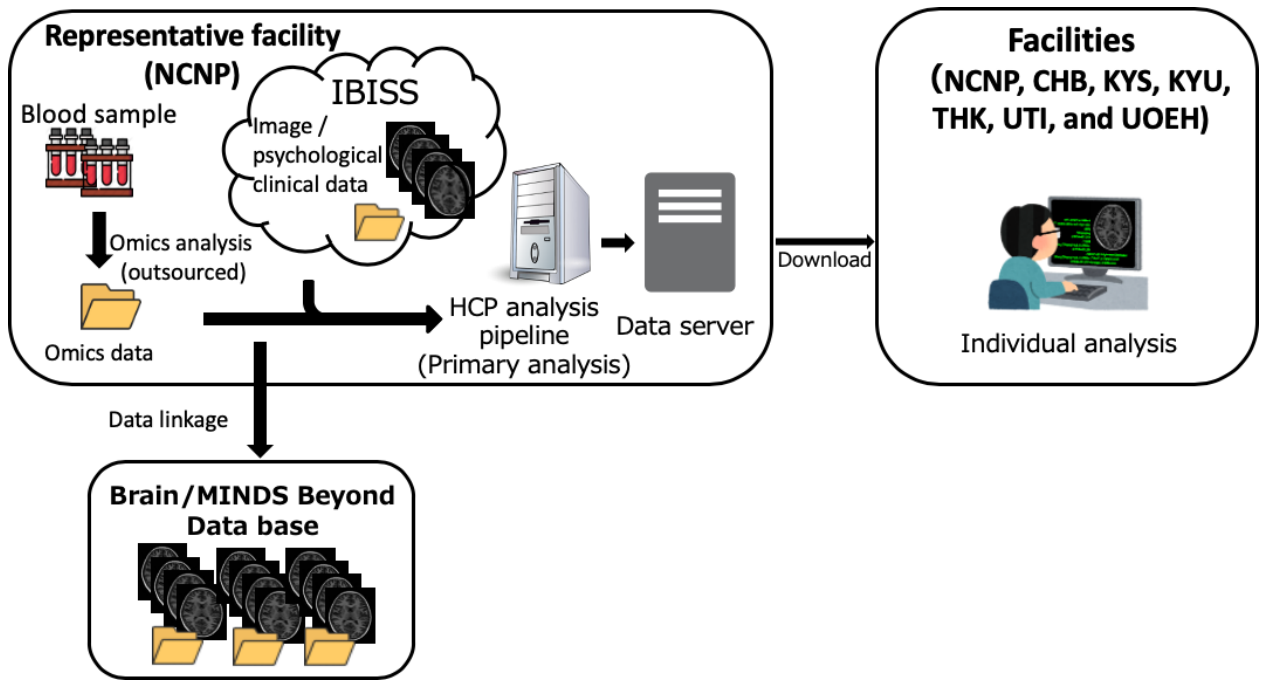

Figure 1 Data logistics. CHB, Chiba University; HCP, Human Connectome Project; IBISS, Integrative Brain Imaging Support System; KYS, Kyushu University; KYU, University of Kyoto; NCNP, National Center of Neurology and Psychiatry; THK, Tohoku University; UOEH, University of Occupational and Environmental Health; UTI, The University of Tokyo IRCN.

calculated based on interpersonal therapy $(\mathrm{n}=65$, mean $=2.37$, $\mathrm{SD}=1.25)$ versus $\mathrm{CBT}(\mathrm{n}=65$, mean $=1.57, \mathrm{SD}=1.25),{ }^{18}$ a single group of 27 cases is required based on a comparison of average outcomes of two independent samples at the time of postintervention (t-test); two tails, $\alpha=0.05,1-\beta=0.80$. Hence, a total of 54 patients is required (27 cases for $\mathrm{AN}$; 27 cases for $\mathrm{BN})$. We plan to complete 72 cases among the joint research facilities. For 5 years, considering the dropout rate of CBT for ED treatment (about 40\%) and the consent rate for MRI (about 60\%), and differences in CBT for each facility, we plan to recruit 200 patients with EDs who are introduced to CBT at all facilities. We expect 120 cases to obtain consent for MRI examination and 72 cases to complete CBT. Regarding the disease type, the number of patients with $\mathrm{AN}$ and $\mathrm{BN}$ is expected to be about 1:1 in the end. Tohoku University and Kyoto University have mainly implemented CBT for patients with BN; conversely, Tokyo University, University of Occupational and Environmental Health and Kyushu University have mainly implemented CBT for patients with AN, whereas NCNP and Chiba University have implemented CBT for patients with $\mathrm{AN}$ and $\mathrm{BN}$ almost equally. Furthermore, we will collect 70 cases in the healthy control group. In particular, in order to verify the effects of low body weight, we will collect data for 30 healthy women with a BMI between 16 and 17.5 (BMI values satisfy one of the diagnoses of $\mathrm{AN}$ ) and no ED pathology.

\section{Trial status}

The first participant was included in this study on 16 June 2020. The end of the recruitment phase is currently scheduled for 31 July 2024.

\section{ETHICS AND DISSEMINATION}

\section{Data distribution}

At the end of the project period, the data will be publicly distributed to researchers through public databases. All imaging, demographic and clinical data are shared between the participating sites and will be made publicly available in 2024. To the best of our knowledge, this is one of the first multisite human brain MRI projects investigating multiple mental and neurological disorders throughout life. The Brain/MINDS Beyond human brain MRI project will help identify common and diseasespecific pathophysiological features of brain disease and develop imaging biomarkers for clinical practice.

\section{Ethical regulation}

We follow the ethical regulation of the previous study. ${ }^{85}$ Sharing neuropsychiatric patient data that can contain information linked to subjects' privacy requires special attention. ${ }^{94}$ Hence, the Brain/MINDS Beyond project has made NCNP the core site for supporting ethical considerations. Before participating in the project, all institutions are required to have their research plans approved by their ethical review committee. This includes the following points and ethical documentation: (1) MR images and clinical data of the participants can be shared within the Brain/MINDS Beyond Project or Japanese/ international scientific institutions. Anonymised MR images with limited clinical data may become publicly available on an open database for research purposes, (2) MR images of the participants may be compared with nonhuman primate MRI data and (3) the intellectual property rights derived from the research of the Brain/MINDS Beyond project shall be attributed to the researcher's institute, not the participants. All participants, after receiving the full description of the experiment, are required to provide written informed consent to participate in this project. The Japanese regulations for the sharing of personal information used for research purposes require attention when handling two types of data: 'individual identification codes' and 'special carerequired personal information' (http://www.japanese lawtranslation.go.jp/law $/$ detail $/$ ? id $=2781 \& \mathrm{vm}=04 \& \mathrm{re}=$ 
01). The individual identification code is a direct identifier and is sufficient to identify a particular individual. Special care-required personal information represents indirect identifiers that need special care in handling in order to avoid potential disadvantages to the participants. In consideration of these regulations, data accompanied with the MR images are limited in the publicly accessible open database and include only 5-year age bins, sex, diagnostic information, handedness, simple socioeconomic status, clinical scale scores and sleepiness scale scores. In the Brain/MINDS Beyond project, we exclude the datasets of MR images containing facial information from the data in the publicly accessible open database.

\section{DISCUSSION}

The strengths of this research are listed below. First, we aimed to construct a brain image database of EDs using the longitudinal brain imaging scans of ED patients from multiple facilities. To date, brain imaging research for ED has been limited to small-scale cross-sectional studies. ${ }^{36-39}$ For this reason, it is important to establish a brain imaging database of EDs using brain images from ED patients across multiple facilities and longitudinally to identify clinically useful diagnostic and therapeutic markers and prognostic predictors. Second, data collected before and after CBT treatment will be collected longitudinally, and treatment responsiveness can be input as a variable. In addition, it is possible to explore the effects of treatment responsiveness by treating childhood trauma as a treatment resistance factor and covariate. Third, the machine learning method can be used to develop into analytical research that leads to an integrated understanding of the pathology of EDs and even neuropsychiatric disorders. Thus far, studies that apply machine learning techniques to brain imaging data from patients with ED have begun to gradually appear but remain limited to cross-sectional studies using samples of 15-24 patients in a single group. ${ }^{95}{ }^{96}$ To date, there remains no longitudinal data from before and after treatment and the association with other clinical markers. Lastly, we will apply an omics analysis to identify clinical markers of ED. Thus far, previous studies have been reported in Japan addressing the susceptibility gene for feeding regulators: the relationship between Ghrelin polymorphism and $\mathrm{BN}^{97}$; the association with young female dissatisfaction of the body, physique and high-density lipoprotein cholesterol $^{98}$; and the association with AN and the fatty acid amide hydrolase (FAAH) polymorphism. ${ }^{99}$ A genomewide correlation analysis (GWAS) using the world's first microsatellite marker for AN was performed, and single nucleotide polymorphisms (SNPs) showing AN sensitivity were identified in at least three gene regions (exon 9 of the CNTN5 gene, the 3 -downstream region of the SPATA17 gene and TOX3 gene). ${ }^{100}$ Furthermore, in a GWAS using SNP markers, a previous study has suggested that having the minor $385 \mathrm{~A}$ allele of the FAAH gene may be protective against restricting AN. Using the knowledge of ED genetic research, it is possible to narrow down the genes targeted in an omics analysis for ED carried out in this research project. Omics research for EDs has only been reported in a cross-sectional study of a few groups for $\mathrm{AN},{ }^{101}$ and no longitudinal study before and after treatment including CBT has been conducted. ED omics research is now beginning to gain traction. Hence, we will design to handle multivariates that includes omics analysis results in brain imaging data. We reduced the dimensions using deep learning techniques and will perform a multiple regression analysis using machine learning algorithms. This is expected to generate evidence for clinical markers of EDs (diagnostic markers, therapeutic effects and therapeutic response prediction markers).

This study has some limitations. First, the number of participants by sites may not be uniform depending on the recruitment of patients and other situations. Also, the number of total participants is too small, and therefore, it is not possible to use machine learning approaches to explore appropriate models without overfitting the available data. Second, although harmonisation is applied, the measurement variability may increase because MRI scanners and imaging protocols can differ between manufacturers. For example, an image scanned using the SRPB imaging protocol has a lower signal-to-noise ratio and resolution compared with an image scanned using HARP. Also, because of the presence or absence of the multiband option, the sampling rate can be up to three times different among facilities. Despite these limitations, such challenging and exploratory approaches are necessary to construct a brain imaging database of patients with EDs from multiple institutions as a first step. Hopefully, by sharing data between other research teams who are collecting brain imaging data on patients with EDs, we will be able to collect a sufficient amount of data.

\section{Author affiliations}

${ }^{1}$ Research Center for Child Mental Development, Chiba University, Chiba, Japan

${ }^{2}$ Japan Society for the Promotion of Science, Tokyo, Japan

${ }^{3}$ United Graduate School of Child Development, Osaka University, Kanazawa University, Hamamatsu University School of Medicine, Chiba University and University of Fukui, Chiba, Japan

${ }^{4}$ Department of Behavioral Medicine, National Institute of Mental Health, National Center of Neurology and Psychiatry, Tokyo, Japan

${ }^{5}$ Department of Psychiatry, Graduate School of Medicine, Kyoto University, Kyoto, Japan

${ }^{6}$ Division of Psychosomatic Medicine, Department of Neurology, University of Occupational and Environmental Health, Kitakyushu, Japan

${ }^{7}$ Department of Psychosomatic Medicine, Graduate School of Medical Sciences, Kyushu University, Fukuoka, Japan

${ }^{8}$ Department of Psychosomatic Medicine, Tohoku University Hospital, Sendai, Japan

${ }^{9}$ Department of Stress Sciences and Psychosomatic Medicine, Graduate School of Medicine, The University of Tokyo, Tokyo, Japan

${ }^{10}$ Department of Radiology, University of Occupational and Environmental Health

School of Medicine, Kitakyushu, Japan

${ }^{11}$ Department of Psychosomatic Medicine, Kitakyushu Municipal Medical Center,

Fukuoka, Japan

${ }^{12}$ Department of Behavioral Medicine, Tohoku University Graduate School of Medicine, Sendai, Japan

${ }^{13}$ Department of Cognitive Behavioral Physiology, Graduate School of Medicine, Chiba University, Chiba, Japan 
Acknowledgements We are very grateful to the patients for participating in the trial dissemination. We would also like to thank the authors of this study and our colleagues supporting the study.

Contributors $\mathrm{SH}, \mathrm{YH}$ and NK participated in the design, drafted and modified the manuscript. YH, MI, NK, KazufY, YM, TA, KazuhY and YS are the coinvestigators of the study who obtained funding and significantly contributed to the conception or design. AyS, YE, JT, NN, TT, HH, TN, KT, KW, HA, MG, ST, SF and ES participated in the design and substantially contributed to the different stages of the study's development towards its practical conduction. AtS is the principal investigator of the study who obtained funding, modified the manuscript and provided substantial contributions to the study conception and design. All authors read and approved the final manuscript.

Funding This work was supported by AMED under grant number JP20dm0307104. Competing interests None declared.

Patient consent for publication Not required.

Supplemental material This content has been supplied by the author(s). It has not been vetted by BMJ Publishing Group Limited (BMJ) and may not have been peer-reviewed. Any opinions or recommendations discussed are solely those of the author(s) and are not endorsed by BMJ. BMJ disclaims all liability and responsibility arising from any reliance placed on the content. Where the content includes any translated material, BMJ does not warrant the accuracy and reliability of the translations (including but not limited to local regulations, clinical guidelines, terminology, drug names and drug dosages), and is not responsible for any error and/or omissions arising from translation and adaptation or otherwise.

Open access This is an open access article distributed in accordance with the Creative Commons Attribution Non Commercial (CC BY-NC 4.0) license, which permits others to distribute, remix, adapt, build upon this work non-commercially, and license their derivative works on different terms, provided the original work is properly cited, appropriate credit is given, any changes made indicated, and the use is non-commercial. See: http://creativecommons.org/licenses/by-nc/4.0/.

\section{ORCID iDs}

Sayo Hamatani http://orcid.org/0000-0001-7847-6381

Yoshiyuki Hirano http://orcid.org/0000-0003-3844-3061

Hiroaki Adachi http://orcid.org/0000-0002-9302-4663

\section{REFERENCES}

1 American Psychiatric Association,. Diagnostic and statistical manual of mental disorders. Fifth Ed. Washington, DC: American Psychiatric Association Publishing, 2013. ISBN: 9780890425558.

2 Nakai Y, Nin K, Noma S. Eating disorder symptoms among Japanese female students in 1982, 1992 and 2002. Psychiatry Res 2014;219:151-6.

3 Arcelus J, Mitchell AJ, Wales J, et al. Mortality rates in patients with anorexia nervosa and other eating disorders. A meta-analysis of 36 studies. Arch Gen Psychiatry 2011;68:724-31.

4 Fichter MM, Quadflieg N. Mortality in eating disorders - results of a large prospective clinical longitudinal study. Int. J. Eat. Disord. 2016;49:391-401.

5 Herzog DB, Greenwood DN, Dorer DJ, et al. Mortality in eating disorders: a descriptive study. Int J Eat Disord 2000;28:20-6.

6 Papadopoulos FC, Ekbom A, Brandt L, et al. Excess mortality, causes of death and prognostic factors in anorexia nervosa. $\mathrm{Br} J$ Psychiatry 2009;194:10-17.

7 Rosling AM, Sparén P, Norring C, et al. Mortality of eating disorders: a follow-up study of treatment in a specialist unit 1974-2000. Int J Eat Disord 2011;44:304-10.

8 Takakura S, Nozaki T, Nomura Y, et al. Factors related to renal dysfunction in patients with anorexia nervosa. Eat Weight Disord 2006;11:73-7.

9 Facchini M, Sala L, Malfatto G, et al. Low-K+ dependent QT prolongation and risk for ventricular arrhythmia in anorexia nervosa. Int J Cardiol 2006;106:170-6.

10 J.e M, Crow S. Medical complications of anorexia nervosa and Bulimia nervosa. Curr Opin Psychiatry 2006;19:438-43.

11 Jáuregui-Garrido B, Jáuregui-Lobera I. Sudden death in eating disorders. Vasc Health Risk Manag 2012;8:91-8.

12 Westmoreland P, Krantz MJ, Mehler PS. Medical complications of anorexia nervosa and Bulimia. Am J Med 2016;129:30-7.

13 Steinhausen $\mathrm{H}-\mathrm{C}$. The outcome of anorexia nervosa in the 20 th century. AJP 2002;159:1284-93.
14 Keel PK, Brown TA. Update on course and outcome in eating disorders. Int J Eat Disord 2010;43:195-204.

15 Hay PJ, Touyz S, Sud R. Treatment for severe and enduring anorexia nervosa: a review. Aust N Z J Psychiatry 2012;46:1136-44.

16 Fairburn CG. Cognitive behavior therapy and eating disorders. Guilford Press, 2008.

17 American Psychiatric Association. Diagnostic and statistical manual of mental disorders. 5th Edition. Washington: American Psychiatric Association Publishing, 2013.

18 de Jong M, Schoorl M, Hoek HW. Enhanced cognitive behavioural therapy for patients with eating disorders. Curr Opin Psychiatry 2018;31:436-44.

19 Fairburn CG, Cooper Z, Doll HA, et al. Transdiagnostic cognitivebehavioral therapy for patients with eating disorders: a two-site trial with 60-week follow-up. Am J Psychiatry 2009;166:311-9.

20 Fairburn CG, Bailey-Straebler S, Basden S, et al. A transdiagnostic comparison of enhanced cognitive behaviour therapy (CBT-E) and interpersonal psychotherapy in the treatment of eating disorders. Behav Res Ther 2015;70:64-71.

21 National Institute for clinical Excellence (NICE). Eating disorders: recognition and treatment|Guidance and guidelines|NICE. 2017. Available: https://www.nice.org.uk/guidance/ng69/chapter/ Recommendations\#treating-bulimia-nervosa [Accessed 4 July 2020].

22 Hofmann SG, Asnaani A, Vonk IJJ, et al. The efficacy of cognitive behavioral therapy: a review of meta-analyses. Cognit Ther Res 2012;36:1:427-40.

23 Linardon J. Meta-Analysis of the effects of cognitive-behavioral therapy on the core eating disorder maintaining mechanisms: implications for mechanisms of therapeutic change. Cogn Behav Ther 2018;47:107-25.

24 Linardon J, Wade T, de la Piedad Garcia X, Garcia delaP X, et al. Psychotherapy for Bulimia nervosa on symptoms of depression: a meta-analysis of randomized controlled trials. Int $J$ Eat Disord 2017;50:1124-36.

25 Vocks S, Tuschen-Caffier B, Pietrowsky R. Meta-Analysis of the effectiveness of psychological and pharmacological treatments for binge eating disorder. Int J Eat Disord 2010;43:205-17.

26 Linardon J, Wade TD, de la Piedad Garcia X, Garcia delaP X, et al. The efficacy of cognitive-behavioral therapy for eating disorders: a systematic review and meta-analysis. J Consult Clin Psychol 2017;85:1080-94.

27 Setsu R, Asano K, Numata N, et al. A single-arm pilot study of guided self-help treatment based cognitive behavioral therapy for Bulimia nervosa in Japanese clinical settings. BMC Res Notes 2018;11:25:257.

28 Hamatani S, Numata N, Matsumoto K, et al. Internet-Based cognitive behavioral therapy via Videoconference for patients with Bulimia nervosa and Binge-Eating disorder: pilot prospective singlearm feasibility trial. JMIR Form Res 2019;3:e15738:23.

29 Linardon J, Hindle A, Brennan L. Dropout from cognitive-behavioral therapy for eating disorders: a meta-analysis of randomized, controlled trials. Int J Eat Disord 2018;51:381-91.

30 Chen J, Papies EK, Barsalou LW. A core eating network and its modulations underlie diverse eating phenomena. Brain Cogn 2016;110:20-42.

31 Joos AAB, Saum B, van Elst LT, et al. Amygdala hyperreactivity in restrictive anorexia nervosa. Psychiatry Res 2011;191:31:189-95.

32 Friederich $\mathrm{H}-\mathrm{C}$, Walther S, Bendszus M, et al. Grey matter abnormalities within cortico-limbic-striatal circuits in acute and weight-restored anorexia nervosa patients. Neuroimage 2012:59:1106-13.

33 Gaudio S, Nocchi F, Franchin T, et al. Gray matter decrease distribution in the early stages of anorexia nervosa restrictive type in adolescents. Psychiatry Res 2011;191:24-30.

34 Fonville L, Giampietro V, Williams SCR, et al. Alterations in brain structure in adults with anorexia nervosa and the impact of illness duration. Psychol Med 2014;44:1965-75.

35 Frank GK, Shott ME, Hagman JO, et al. Alterations in brain structures related to taste reward circuitry in ill and recovered anorexia nervosa and in Bulimia nervosa. AJP 2013;170:1152-60.

36 Sato Y, Saito N, Utsumi A, et al. Neural basis of impaired cognitive flexibility in patients with anorexia nervosa. PLoS One 2013;8:e61108:10.

37 Isobe M, Kawabata M, Murao E. Exaggerated envy and guilt measured by economic games in Japanese women with anorexia nervosa. Biopsychosoc Med 2018;6:12-19.

38 Kodama N, Moriguchi Y, Takeda A, et al. Neural correlates of body comparison and weight estimation in weight-recovered anorexia nervosa: a functional magnetic resonance imaging study. Biopsychosoc Med 2018;12:15. 
39 Morita C, Tsuji H, Hata T, et al. Gut dysbiosis in patients with anorexia nervosa. PLoS One 2015;10:e0145274:18.

40 Fairburn CG, Cooper Z, O'Connor M. Eating disorder examination (16.0D). In: Fairburn CG, ed. Cognitive behavior therapy and eating disorders. New York: Guilford Press, 2008.

41 Tachi T, Murakami K, Washizuka T. Application of the eating disorder examination (EDE) to Japanese patients with eating disorders: reliability and validity of the Japanese version of EDE. Jpn J Psychosom Med 2005;45:785-92.

42 Fairburn CG, Beglin SJ. Assessment of eating disorders: interview or self-report questionnaire? Int J Eat Disord 1994;16:363-70.

43 Sheehan DV, Lecrubier Y, Sheehan KH. The Mini-International neuropsychiatric interview (M. I.N.I.): the development and validation of a structured diagnostic psychiatric interview for DSMIV and ICD-10. J Clin Psychiatry 1998;59:22-33.

44 Muramatsu K, Miyaoka H, Kamijima K, et al. The patient health questionnaire, Japanese version: validity according to the mini-international neuropsychiatric interview-plus. Psychol Rep 2007;101:952-60.

45 Matsuoka K, Uno M, Kasai K, et al. Estimation of premorbid IQ in individuals with Alzheimer's disease using Japanese ideographic script (Kanji) compound words: Japanese version of National Adult Reading Test. Psychiatry Clin Neurosci 2006;60:332-9.

46 Nelson H, Willison J. National Adult Reading Test (NART).. In: Windsor UK: NFER-Nelson, ed. 2Nd, 1991.

47 Kojima M, Furukawa TA, Takahashi $\mathrm{H}$, et al. Cross-Cultural validation of the Beck depression Inventory-II in Japan. Psychiatry Res 2002;110:31:291-9.

48 Beck A, Steer R, Brown G. BDI-II: Beck Depression Inventory. Second Edition. San Antonio, TX: The Psychological Corporation, 1996.

49 Hidano T, Fukuhara M, Iwawaki S. Shin-ban STAI Manyuaru [New STAI Manual State-trait Anxiety Inventoryform JYZ. Tokyo, Japan: Jitsumu Kyoiku Shuppan (in Japanese), 2000.

50 Spielberger CD, Gorsuch RL, Lushene R. Manual for the State-Trait Anxiety Inventory. Palo Alto, CA: Consulting Psychologists Press, 1983.

51 CostaTPJr, McCrae RR. The NEO-PI/NEO-FFI manual supplement. Odessa, FL: Psychological Assessment Resources, 1989.

52 Shimonaka Y, Nakazato K, Gondo Y. NEO-PI-R, NEO-FFI manual for the Japanese version revised and enlarged edition. Tokyo: Tokyo Shinri, Inc, 2011.

53 Bagby RM, Parker JDA, Taylor GJ. The twenty-item Toronto Alexithymia scale-I. item selection and cross-validation of the factor structure. J Psychosom Res 1994;38:23-32.

54 Komaki G, Maeda M, Arimura T. The reliability and factorial validity of the Japanese version of the 20-Item Toronto Alexithymia scale (TAS-20). Japanese Journal of Psychosomatic Medicine 2003;43:839-46.

55 bell S. Adult version facial expression recognition test. Available: http://www.saccess55.co.jp/untitled406.html [Accessed 4 July 2020].

56 Oldfield RC. The assessment and analysis of handedness: the Edinburgh inventory. Neuropsychologia 1971;9:97-113.

57 Hatta T, Hotta C. Which Inventory should be used to Assess Japanese Handedness : Comparison between Edinburgh and H. N. Handedness Inventories. Journal of Human Environmental Studies 2008;6:45-8.

58 Bernstein DP, Ahluvalia T, Pogge D, et al. Validity of the childhood trauma questionnaire in an adolescent psychiatric population. Journal of the American Academy of Child \& Adolescent Psychiatry 1997;36:340-8.

59 Mizuki R, Fujiwara T. Validation of the Japanese version of the childhood trauma Questionnaire-Short form (CTQ-J). Psychological Trauma: Theory, Research, Practice, and Policy.

60 Foa EB, Cashman L, Jaycox L, et al. The validation of a self-report measure of posttraumatic stress disorder: the posttraumatic diagnostic scale. Psychol Assess 1997;9:445-51.

61 Itoh M, Ujiie Y, Nagae N, et al. The Japanese version of the posttraumatic diagnostic scale: validity in participants with and without traumatic experiences. Asian J Psychiatr 2017;25:1-5.

62 Ohara C, Sekiguchi A, Takakura S, et al. Effectiveness of enhanced cognitive behavior therapy for Bulimia nervosa in Japan: a randomized controlled trial protocol. Biopsychosoc Med 2020;14:2.

63 Epstein NB, Baldwin LM, Bishop DS. The McMaster family assessment DEVICE*. J Marital Fam Ther 1983;9:171-80.

64 Saeki T, Asukai N, Miyake Y. Reliability and validity of the Japanese version of the family assessment device (FAD). Arch Psychiatr Diagn Clin Eval 1997;8:181-92.
65 Tamura S, Ishikuma T, Preferences H-S. And burnout: junior high school teachers in Japan. Japanese Journal of educational psychology 2006;54:75-89.

66 Mehling WE, Price C, Daubenmier JJ, et al. The multidimensional assessment of interoceptive awareness (Maia). PLoS One 2012; 7:e48230.

67 Shoji M, MehlingEW HM, et al. Investigating multidimensional interoceptive awareness in a Japanese population: validation of the Japanese MAIA-J. Front Psychol 1855;2018:9.

68 Patton JH, Stanford MS, Barratt ES. Factor structure of the barratt impulsiveness scale. J Clin Psychol 1995;51:768-74.

69 Someya T, Sakado K, Seki T, et al. The Japanese version of the Barratt Impulsiveness scale, 11th version (BIS-11): its reliability and validity. Psychiatry Clin Neurosci 2001;55:111-4.

70 Tadai T, Nakamura M, Okazaki S, et al. The prevalence of obsessive-compulsive disorder in Japan: a study of students using the Maudsley Obsessional-Compulsive inventory and DSM-III-R. Psychiatry Clin Neurosci 1995;49:39-41.

71 Hodgson RJ, Rachman S. Obsessional-compulsive complaints. Behav Res Ther 1977;15:389-95.

72 Wakabayashi A, Tojo Y, Baron-Cohen S, et al. TThe AutismSpectrum Quotient (AQ) Japanese version: evidence from highfunctioning clinical group and normal adults]. Shinrigaku Kenkyu 2004;75:78-84.

73 Baron-Cohen S, Wheelwright S, Skinner R, et al. The autismspectrum quotient (AQ): evidence from Asperger syndrome/ high-functioning autism, males and females, scientists and mathematicians. J Autism Dev Disord 2001;31:5-17.

74 Rosenberg M. Society and adolescent self-image. New Jersey: Princeton University Press, 1965.

75 Mimura C, Griffiths P. A Japanese version of the Rosenberg self-esteem scale: translation and equivalence assessment. $J$ Psychosom Res 2007;62:589-94.

76 Kamimura E, Ebihara Y, Sato K. A validation of three dimen sional model of coping response and development of the Tri-axial coping scale (TAC-24). Bulletin of Counseling and School Psychology 1995;33:41-7.

77 Tracey TJ, Kokotovic AM. Factor structure of the working alliance inventory. Psychol Assess 1989;1:207-10.

78 Tsuchiya A, Ikeda S, Ikegami N, et al. Estimating an EQ5D population value set: the case of Japan. Health Econ 2002;11:341-53.

79 EuroQol Group. EuroQol - a new facility for the measurement of health-related quality of life. Health Policy 1990;16:199-208.

80 Spitzer RL, Kroenke K, Williams JB. Validation and utility of a selfreport version of PRIME-MD: the PHQ primary care study. JAMA 1999:282:10:1737-44.

81 Muramatsu K. Questionnaire pH (PHQ-9, PHQ-15) Japanese version and generalized anxiety Disorder-7 Japanese version up to date. Stud Clin Psychol 2014;7:35-9.

82 Spitzer RL, Kroenke K, Williams JBW, et al. A brief measure for assessing generalized anxiety disorder: the GAD-7. Arch Intern Med 2006;166:22:1092-7.

83 Psychiatric Research Unit. Who collaborating center for mental health, WHO-5 questionnaires. Available: https://www.psykiatriregionh.dk/who-5/who-5-questionnaires/Pages/default.aspx [Accessed 4 July 2020].

84 Hoddes E, Zarcone V, Smythe H, et al. Quantification of sleepiness: a new approach. Psychophysiology 1973;10:431-6.

85 Koike S, Tanaka SC, Okada T. Brain/MINDS beyond human brain MRI study: multi-site harmonization for brain disorders throughout the lifespan. bioRxiv 2020

86 Whitfield-Gabrieli S, Nieto-Castanon A. Conn : A Functional Connectivity Toolbox for Correlated and Anticorrelated Brain Networks. Brain Connect 2012;2:125-41.

87 Fortin J-P, Cullen N, Sheline YI, et al. Harmonization of cortical thickness measurements across scanners and sites. Neuroimage 2018;167:104-20.

88 Fortin J-P, Parker D, Tunç B, et al. Harmonization of multi-site diffusion tensor imaging data. Neuroimage 2017;161:149-70.

89 Johnson WE, Li C, Rabinovic A. Adjusting batch effects in microarray expression data using empirical Bayes methods. Biostatistics 2007;8:118-27.

90 Pedregosa F, Varoquaux G, Gramfort A. Scikitlearn: machine learning in python. J Mach Learn Res 2011;12:2825-30.

91 Ambroise C, McLachlan GJ. Selection bias in gene extraction on the basis of microarray gene-expression data. Proc Natl Acad Sci U $S$ A 2002;99:6562-6.

92 Cawley GC, Talbot NLC. On over-fitting in model selection and subsequent selection bias in performance evaluation. J Mach Learn Res 2010;11:2079-107. 
93 ten Kate M, Redolfi A, Peira E, et al. Mri predictors of amyloid pathology: results from the EMIF-AD multimodal biomarker discovery study. Alzheimers Res Ther 2018;10:100.

94 Sadato N, Morita K, Kasai K, et al. Neuroethical issues of the Brain/ MINDS project of Japan. Neuron 2019;101:385-9.

95 Lavagnino L, Amianto F, Mwangi B, et al. Identifying neuroanatomical signatures of anorexia nervosa: a multivariate machine learning approach. Psychol Med 2015;45:2805-12.

96 Lavagnino L, Mwangi B, Cao B, et al. Cortical thickness patterns as state biomarker of anorexia nervosa. Int $\mathrm{J}$ Eat Disord 2018;51:241-9.

97 Ando T, Komaki G, Naruo T, et al. Possible role of preproghrelin gene polymorphisms in susceptibility to Bulimia nervosa. Am. J. Med. Genet. 2006;141B:5:929-34.

98 Ando T, Ichimaru Y, Konjiki F, et al. Variations in the preproghrelin gene correlate with higher body mass index, fat mass, and body dissatisfaction in young Japanese women. Am J Clin Nutr 2007:86:25-32.

99 Ando T, Tamura N, Mera T. Association of the c.385C $>A$ (p.Pro129Thr) polymorphism of the fatty acid amide hydrolase gene with anorexia nervosa in the Japanese population.; Japanese Genetic Research Group For Eating Disorders. Mol Genet Genomic Med 2014;2:313-8.

100 Nakabayashi K, Komaki G, Tajima A. Japanese genetic research Group for eating disorders (JGRED), Shirasawa S. identification of novel candidate loci for anorexia nervosa at 1q41 and 11q22 in Japanese by a genome-wide association analysis with microsatellite markers. J Hum Genet 2009;54:531-7.

101 Föcker M, Timmesfeld N, Scherag S, et al. Comparison of metabolic profiles of acutely ill and short-term weight recovered patients with anorexia nervosa reveals alterations of 33 out of 163 metabolites. J Psychiatr Res 2012;46:1600-9. 